\title{
Erratum to: Clinical efficiency of Piezo-ICSI using micropipettes with a wall thickness of $0.625 \mu \mathrm{m}$
}

\author{
Kenichiro Hiraoka $^{1} \cdot$ Seiji Kitamura ${ }^{2}$
}

Published online: 27 February 2016

(C) Springer Science+Business Media New York 2016

Erratum to: J Assist Reprod Genet (2015) 32:1827-1833

DOI 10.1007/s10815-015-0597-9

The authors would like to apologize for accidentally making a mistake in the inner and outer diameter calculation of the Piezo-ICSI micropipettes.

\begin{tabular}{llll}
\hline Micropipette & & Wrong & Right \\
\hline PIN07-20FT & Inner diameter & $5 \mu \mathrm{m}$ & $5.25 \mu \mathrm{m}$ \\
& Wall thickness & $0.925 \mu \mathrm{m}$ & $0.875 \mu \mathrm{m}$ \\
\multirow{2}{*}{ PINU06-20FT } & Inner diameter & $5 \mu \mathrm{m}$ & $5.1 \mu \mathrm{m}$ \\
& Wall thickness & $0.625 \mu \mathrm{m}$ & $0.45 \mu \mathrm{m}$ \\
\hline
\end{tabular}

The online version of the original article can be found at http://dx.doi.org/ 10.1007/s10815-015-0597-9.

\section{Kenichiro Hiraoka}

hiraoka@oct.email.ne.jp

1 Kameda Medical Center, 929 Higashi-cho,

Kamogawa-shi, Chiba 296-8602, Japan

2 Ogikubo Hospital Niji Clinic, 4-32-2-8F9F Ogikubo, Suginami-ku, Tokyo 167-0051, Japan 